\title{
A NUMERICAL STUDY FOR GROWING SANDPILES ON FLAT TABLES WITH WALLS
}

\author{
M. Falcone ${ }^{1}$ and S. Finzi Vita ${ }^{1}$ \\ ${ }^{1}$ Università di Roma La Sapienza, Dipartimento di Matematica, Roma, Italy, \\ $\{$ falcone,finzi $\} @$ mat.uniromal.it ${ }^{*}$
}

\begin{abstract}
We continue our study on the approximation of a system of partial differential equations recently proposed by Hadeler and Kuttler to model the dynamics of growing sandpiles on a flat bounded table. The novelty here is the introduction of (infinite) walls on the boundary of the domain and the corresponding modification of boundary conditions for the standing and for the rolling layers. An explicit finite difference scheme is introduced and new boundary conditions are analyzed. We show experiments in $1 \mathrm{D}$ and 2D which characterize the steady-state solutions.
\end{abstract}

keywords: granular matter, hyperbolic systems, finite differences schemes

\section{Introduction}

In this paper we continue our study on numerical methods for the simulation of growing sandpiles, started in [5] and [7] for the so-called open table problem (we refer to those papers for a list of recent references on the modelling of granular matter). The prototype situation is the following. A flat bounded table (a bounded open domain $\Omega$ of $\mathbb{R}^{2}$ ) is initially empty and the sand is poured on a subset of $\Omega$ according to the value of a nonnegative function $f(x, t)$ (the source). The pile grows in height with a slope which is always lower than a characteristic value, which naturally depends on the the physical properties of the granular matter.

Among the pde-models recently studied for the description of this phenomenon (see for example $[1,3,8]$ ), we will focus our attention on the one proposed by Hadeler and Kuttler [8], based on two interacting variables: the standing layer $u$ and the rolling layer $v$. The relations between $u$ and $v$ are

\footnotetext{
*Paper written with financial support of the MIUR Project COFIN 2003 "Modellistica Numerica per il Calcolo Scientifico ed Applicazioni Avanzate"
}

Please use the following format when citing this chapter:

Falcone, M., and Finzi Vita, S., 2006, in IFIP International Federation for Information Processing, Volume 202, Systems, Control, Modeling and Optimization, eds. Ceragioli, F., Dontchev, A., Furuta, H., Marti, K., Pandolfi, L., (Boston: Springer), pp. 127-137. 
described by the system of nonlinear partial differential equations

$$
\begin{array}{cl}
v_{t}=\nabla \cdot(v \nabla u)-(1-|\nabla u|) v+f, & \text { in } \Omega \times(0, T) \\
u_{t}=(1-|\nabla u|) v, & \text { in } \Omega \times(0, T) \\
u(\cdot, t)=0 \quad \text { on } \partial \Omega, \quad u(\cdot, 0)=0 & \text { in } \Omega .
\end{array}
$$

It is important to note that existence and uniqueness results are difficult to prove even when the table is "open", i.e. when the sand can fall down from every point of the boundary $\partial \Omega$ and we have the homogeneous boundary condition (3). However, in that situation, a partial characterization of the stationary solutions of that system has been recently given by Cannarsa and Cardialiaguet [2]. In [5] we proposed an approximation scheme for the above system analyzing its properties in the one dimensional case and showing that the scheme mimics several characteristics of the continuous model. More recently Finzi Vita [7] has compared this model with other approaches proposed by Aronsson, Evans and Wu [1] and by Prigozhin [10] and has developed several tests with the three approaches. It is interesting to note that although the three models have theoretically the same set of admissible equilibria the evolution in time of the piles is rather different.

In this paper we want to study a model for a growing pile on a table $\Omega$ whose boundary $\partial \Omega$ can be split into two parts: $\Gamma_{0}$ which is the subset of the boundary where the sand can fall down the table and $\Gamma_{1}$ which is the subset of the boundary where the sand is blocked by a wall. This corresponds to the assumption that the walls are sufficiently high to guarantee that the pile is not trespassing it (for simplicity let us assume that they have infinite height). In this situation we have to impose mixed boundary conditions on $\partial \Omega=\Gamma_{0} \cup \Gamma_{1}$. The boundary condition on $\Gamma_{0}$ is trivial and corresponds to the usual homogeneous Dirichlet boundary condition on $u$ in (3). The boundary condition on $\Gamma_{1}$ deserves some analysis and this will be done later in this paper. Also for the model with walls it is interesting to study the steady-state solutions of the two-layer model and several questions arise: what is the equilibrium configuration (if any) for $u$ and $v$ ? is there any representation formula for them? what is the singular set for $u$ ? In this paper we will try to answer some of these questions, making use of the scheme introduced in [5] (modified with a suitable boundary conditions at the walls), also showing that such modified scheme produces reasonable results which seem to match the physical behavior of the growing piles. A partial analysis of the equilibrium configurations for the same problem (called the tray problem) as well as for the obstacle problem when the table is a surface $z=g(x, y)$ can be found in [9]. However, the main emphasis there is on the equivalence of different types of solutions with the maximal volume solution. 


\section{The two-layers model for the open table}

Following the fundamental theories of de Gennes [3] on granular matter, Hadeler and Kuttler [8] introduced a system of two partial differential equations to govern the growth of a sandpile, seen as the superposition of two distinct layers, the standing and the rolling ones. In their model, an eikonal type equation for the standing layer $u$ forming heaps and slopes is coupled to an advection type equation for the small rolling layer $v$ running down the slope. This leads to system (1)-(2), which has to be complemented with the initial and the Dirichlet boundary conditions (3).

Note that for this model positive rolling layers are allowed during the evolution even before the corresponding standing layer becomes critical. The equilibria $(\bar{u}, \bar{v})$ of (1)-(3)for a constant in time source $f$ solve the system

$$
-\nabla \cdot(v \nabla u)=f, \quad(1-|\nabla u|) v=0 \quad \text { in } \Omega,\left.\quad u\right|_{\partial \Omega}=0 .
$$

Solutions of (4) have been recently characterized by Cannarsa and Cardaliaguet [2] for the 2-dimensional problem by means of viscosity solution techniques. In their result the boundary $\partial \Omega$ is assumed to be sufficiently regular. In order to state their main result let us introduce some notations and definitions. Let $d(x)=\inf \{|x-z|: z \in \partial \Omega\}$ be the distance function from the boundary of $\Omega$, let $\mathcal{R}$ be the set of discontinuity of $\nabla d$ (usually called the ridge) and for every $x \in \Omega \backslash \mathcal{R}$ let $k(x)$ denote the curvature of $\partial \Omega$ at the projection of $x$ onto it (note that the projection is unique for every $x$ not belonging to $\mathcal{R}$ ). Finally, let $\tau(x)=\min \{t \geq 0: x+t \nabla d(x) \in \overline{\mathcal{R}}\}$ indicate the so-called normal distance to $\overline{\mathcal{R}}$.

THEOREM 1 Let $\Omega \subset R^{2}$ be a bounded domain with $C^{2}$ boundary and $f \geq 0$ a continuous function in $\Omega$. Then, there exists a solution of system (4) given by the pair $(u, v)$, where $u=d$ in $\Omega, v=0$ in $\mathcal{R}$ and

$$
v(x)=\int_{0}^{\tau(x)} f(x+t \nabla d(x)) \frac{1-(d(x)+t) k(x)}{1-d(x) k(x)} d t, \quad \forall x \in \Omega \backslash \overline{\mathcal{R}} .
$$

Such a solution is unique in the sense that if $\left(u^{\prime}, v^{\prime}\right)$ is another solution, then $v^{\prime}=v$ in $\Omega$ and $u^{\prime}=d$ in $\{x \in \Omega: v>0\}$.

By the above result there exists a unique equilibrium solution $v$ for any given source $f$, and an integral representation formula is given for it outside $\mathcal{R}$ (where $v=0$ ). On the contrary, for $u$ there is no uniqueness, although every solution must coincide with $d$ in the set where $v>0$. Then, if we denote by $D_{f}$ the set of positivity of $f$ in $\Omega$, two distinct cases can occur:

(a) $\mathcal{R} \subset D_{f}$ : the unique possible asymptotic equilibrium for $u$ is essentially 
given by the distance function $d$.

(b) $\mathcal{R}$ is not contained in $D_{f}$ : the model has an equilibrium configuration $\bar{u}$ which also depends on the source intensity (as clearly shown by the numerical experiments discussed in [5]) and for which a rigorous mathematical characterization is not known. In this situation, that equilibrium configuration is larger then the one predicted by the model by Prygozhin [10], whereas in the first situation they coincide.

In [5] we have proposed a finite difference approximation for the system (1)-(3) in the one dimensional case, which for $\Omega=(0,1)$ corresponds to

$$
\begin{gathered}
v_{t}=\left(v u_{x}\right)_{x}-\left(1-\left|u_{x}\right|\right) v+f=v u_{x x}+v_{x} u_{x}-\left(1-\left|u_{x}\right|\right) v+f \\
u_{t}=\left(1-\left|u_{x}\right|\right) v \\
u(0, t)=u(1, t)=0, \quad u(x, 0)=0 \quad x \in \Omega .
\end{gathered}
$$

Now let us consider the space grid $x_{i}=(i-1) h$, for $i=1, . ., N$ and $h=$ $\Delta x=1 /(N-1)$. We denote by $D^{+} u_{i}$ and $D^{-} u_{i}$ respectively the right and the left discrete derivative for $u$ at $x_{i}$.

Then we discretize the derivative of $u$ at a node $x_{i}$ by the difference with maximal absolute value, namely $D u_{i} \equiv \operatorname{maxmod}\left(D^{-} u_{i}, D^{+} u_{i}\right)$, whereas the corresponding derivative of $v$ is approximated by the upwind (with respect to $D u_{i}$ ) finite difference, i.e.

$$
\bar{D} v_{i} \equiv\left\{\begin{array}{cc}
D^{+} v_{i} & \text { if } D u_{i}>0, D^{+} u_{i}>0 \\
D^{-} v_{i} & \text { if } D u_{i}<0, D^{-} u_{i}<0 \\
0 & \text { otherwise }
\end{array}\right.
$$

We discretize time by a fixed step $\Delta t$ and use the standard notation, where $z_{i}^{n}$ denotes the approximate value of a function $z(x, t)$ for $t=t_{n} \equiv n \Delta t$ and $x=x_{i}$. Using backward Euler for the time derivatives, we obtain our fully explicit scheme for the solution of (6)-(8)

$$
\begin{gathered}
v_{i}^{n+1}=v_{i}^{n}+\Delta t\left[v_{i}^{n} D^{2} u_{i}^{n}+\bar{D} v_{i}^{n} D u_{i}^{n}-\left(1-\left|D u_{i}^{n}\right|\right) v_{i}^{n}+f_{i}\right] \\
u_{i}^{n+1}=u_{i}^{n}+\Delta t\left(1-\left|D u_{i}^{n}\right|\right) v_{i}^{n} \\
u_{i}^{0}=v_{i}^{0}=0 \quad \forall i, \quad u_{1}^{n}=u_{N}^{n}=0 \quad \forall n,
\end{gathered}
$$

where $D^{2} u_{i}^{n}$ denotes the classical 3-point discretization of the second derivative $u_{x x}$ at $x_{i}$. In [5], where several experiments have been reported, it has been proved that the discrete model mimics some properties of the continuous model if the Courant number $\lambda=\Delta t / \Delta x$ is sufficiently small. Namely, the approximation $u^{n}$ is always nonnegative and monotonically increasing in time, the approximation $v^{n}$ stays nonnegative and the bound $\left|D u^{n}\right| \leq 1$ is always satisfied. Moreover, using standard arguments one can show that the above scheme is consistent with the continuous system and that the local error is $O(\Delta x, \Delta t)$. 


\section{The two-layers model with walls}

As we said in the introduction, the main difference with respect to the original model of the open table in [8] is the introduction of the wall on a subset of the boundary. Let us consider the one dimensional case and take $\Omega=(0,1)$, open at the left-hand side and with an infinite wall at $x=1$, i.e. $\Gamma_{0}=\{0\}$ and $\Gamma_{1}=\{1\}$. In order to derive the correct boundary condition on $\Gamma_{1}$, we remark that at the equilibrium configuration we must have

$$
\begin{gathered}
0=\frac{d}{d t} \int_{0}^{1}(\bar{u}+\bar{v}) \mathrm{d} x=\int_{0}^{1}\left(\bar{u}_{t}+\bar{v}_{t}\right) \mathrm{d} x= \\
=\int_{0}^{1}\left(\bar{v} \bar{u}_{x}\right)_{x} \mathrm{~d} x+\int_{0}^{1} f \mathrm{~d} x=\bar{v}(1) \bar{u}_{x}(1)-\bar{v}(0) \bar{u}_{x}(0)+\int_{0}^{1} f \mathrm{~d} x,
\end{gathered}
$$

where we have used (6) and (7) to obtain (13). Note that the last two terms in (13) have a precise physical meaning: the second is the sand falling down from the table at $x=0$, the third the sand falling on the table from the source in the unit time. If the system is in equilibrium, the incoming sand has to balance the sand leaving the table. Then, the necessary boundary condition to be satisfied at $x=1$ will be the complementarity equation

$$
\bar{v}(1) \bar{u}_{x}(1)=0 .
$$

Then, reasoning as in [8], it is easy to determine explicit formulas for the equilibria of such modified problem. If we denote by $D_{f}=\left[x_{1}, x_{2}\right]$ the support of the source term, we can distinguish between two cases:

(a) $x_{2}=1$ ( $D_{f}$ touches the wall): the unique possible asymptotic equilibrium for $u$ is essentially given by the distance function from $\Gamma_{0}$, and we have

$$
u(x)=x, \quad v(x)=\int_{x}^{1} f(z) d z, \quad \forall x \in \Omega .
$$

(b) $x_{2}<1$ (no sand flows along the wall): then the equilibria are uniquely determined in $\left[0, x_{2}\right]$, and we have

$$
u(x)=x, \quad v(x)=\int_{x}^{x_{2}} f(z) d z, \quad \forall x \in\left[0, x_{2}\right],
$$

whereas in $\left(x_{2}, 1\right]$ we can only say that $v=0$. The system is not able to characterize $u$ where $v=0$.

We see that condition (14) is satisfied in both cases simply because $v(1)=0$, but such reduced condition would not be the right one for the dynamic model, since it would block the growth of $u$ at the wall (see (7)). We have then 
considered (14) as the boundary condition on $\Gamma_{1}$ for the evolutive two-layers system, even if this is not the only possible choice.

From the numerical point of view, we had to modify the scheme (9)-(11) at $x_{N}$ in order to implement the wall boundary condition (14). We tried various ways to implement that condition, some of them unsatisfactory:

- If $D u_{N}^{n}>0$ then $v_{N}^{n}=0$. When $x_{2}=1$ the scheme finds the correct equilibria, but the growth process is not uniform near the wall. When $x_{2}<1$, the stationary $u$ is linear in $\left(x_{2}, 1\right)$.

- If $D u_{N}^{n}>0$ then $v_{N}^{n}=0$, else $u_{N}^{n}=u_{N-1}^{n}$. The dynamics is now correct, except for the values at the wall in the case $x_{2}=1$.

- If $D u_{N}^{n}>0$ then $v_{N}^{n}=0$, else $u_{N}^{n}=\left(4 u_{N-1}^{n}-u_{N-2}^{n}\right) / 3$. The second order difference now produces correct values on the wall in both cases. The stationary $u$ is nonlinear in $\left(x_{2}, 1\right)$.

We have then used in the tests of Section 4.1 the scheme (9)-(11) replacing the homogeneous Dirichlet boundary condition $u_{N}^{n}=0$ by

$$
\text { if } D u_{N}^{n}>0 \text { then } v_{N}^{n}=0 \text {, else } u_{N}^{n}=\left(4 u_{N-1}^{n}-u_{N-2}^{n}\right) / 3, \quad \forall n,
$$

for different choices of the source support $D_{f}=\left[x_{1}, x_{2}\right]$.

Let us now examine the 2-dimensional model. With similar arguments the right boundary condition at $\Gamma_{1}$ for the equilibria can be derived using Green's theorem, obtaining

$$
\int_{\Gamma_{1}} v \frac{\partial u}{\partial n} d \sigma=0
$$

Again we have chosen for the dynamic model the point-wise condition $v \frac{\partial u}{\partial n}=$ 0 on $\Gamma_{1}$. However, in two dimensions this problem is much more complex than the open table problem. The extremal points of $\Gamma_{1}$ are usually singular (discontinuity) points for the rolling layer, since at those points infinite transport rays meet. Moreover, the ridge set $\mathcal{R}$, i.e. the singular set of the distance function from $\Gamma_{0}$, can change very much from case to case. In general, it is the union of several arcs which can reach the boundary at the open vertices or at some (regular) points of the wall-boundary $\Gamma_{1}$.

The two-dimensional extension of the scheme (9)-(11) for the open table is straightforward and its main features are preserved. We introduce in the domain $\Omega$ (for simplicity the square $(0,1) \times(0,1))$ an $N \times N$ uniform grid of nodes $x_{i, j}$, and, as usual, we denote by $\left(u_{i, j}^{n}, v_{i, j}^{n}\right)$ the components of the discrete solutions on node $x_{i, j}$ at time $t=t_{n}$. Then, the scheme can be written as

$$
\begin{gathered}
v_{i, j}^{n+1}=v_{i, j}^{n}+\Delta t\left[v_{i, j}^{n} D^{2} u_{i, j}^{n}+\bar{D} v_{i, j}^{n} \cdot D u_{i, j}^{n}\right. \\
\left.-\left(1-\left|D u_{i, j}^{n}\right|\right) v_{i, j}^{n}+f_{i, j}\right]
\end{gathered}
$$




$$
\begin{gathered}
u_{i, j}^{n+1}=u_{i, j}^{n}+\Delta t\left(1-\left|D u_{i, j}^{n}\right|\right) v_{i, j}^{n} \\
u_{i, j}^{0}=v_{i, j}^{0}=0 \quad \forall i, j \\
u_{i, j}^{n}=0 \text { on } \partial \Omega \quad \forall n,
\end{gathered}
$$

where now $D^{2} u_{i, j}$ is the standard five-points discretization of the Laplace operator, and the discrete gradient vectors $D u_{i, j}$ and $\bar{D} v_{i, j}$ are defined, component by component, as in the one-dimensional case.

The application of such scheme to the wall problem requires a modification of boundary conditions. In analogy with the one-dimensional case discussed in the previous section, the boundary condition (16) on $\Gamma_{1}$ is implemented in the scheme by replacing condition (20) with the following ones

$$
\begin{aligned}
& \forall(i, j) \text { such that } x_{i, j} \in \Gamma_{1}: \text { if } D u_{i, j}^{n} \cdot \nu_{i, j}>0 \text { then } v_{i, j}^{n}=0, \\
& \text { else } u_{i, j}^{n}=u_{\left((i, j)-\nu_{i, j}\right)}^{n} \text { and } v_{i, j}^{n}=v_{\left((i, j)-\nu_{i, j}\right)}^{n}, \\
& \forall(i, j) \text { such that } x_{i, j} \in \widetilde{\Gamma}_{0}: u_{i, j}^{n}=0, \\
& \forall \text { vertex } x_{i, j} \in \Gamma_{0}: v_{i, j}^{n}=0,
\end{aligned}
$$

where $\nu_{i, j}$ denotes the outward normal unit vector at $x_{i, j}$. We have found that Neumann boundary conditions both on $u$ and $v$ (see (22)) yield better results in the tests. The technical condition (24) on the open vertices is necessary to avoid locking at those points. When singular points are present on $\Gamma_{1}$, numerical tests show that instability rapidly occurs in time at nodes close to them. In order to prevent this phenomenon we found that it is enough to impose at every time iteration the explicit gradient constraint which is known to hold at the equilibrium:

$$
\left|D u_{i, j}^{n}\right|=\min \left(\left|D u_{i, j}^{n}\right|, 1\right), \quad \forall(i, j), \forall n .
$$

\section{Numerical tests}

\subsection{1-dimensional tests}

For a constant source term $f$ over $D_{f}=\left(x_{1}, x_{2}\right) \subset \bar{\Omega}$, we describe the results of some experiments.

For $x_{2}=1$ (case $(a)$ of Section 3 ), we know that there is only a possible equilibrium, $u(x) \equiv x$ in $\Omega$. In Figure 1 (top) we see two examples of that situation, where the scheme is able to detect the correct dynamics for $u$ and $v$ (circles indicate the profile of the rolling layer). Different values of $x_{1}$ can only influence the final shape of the rolling layer, according to the explicit formula (5). We remark that the first order difference in $u$ becomes strictly positive near the wall, so that the boundary condition (15) of the scheme reduces definitively to $v_{N}^{n}=0$. 

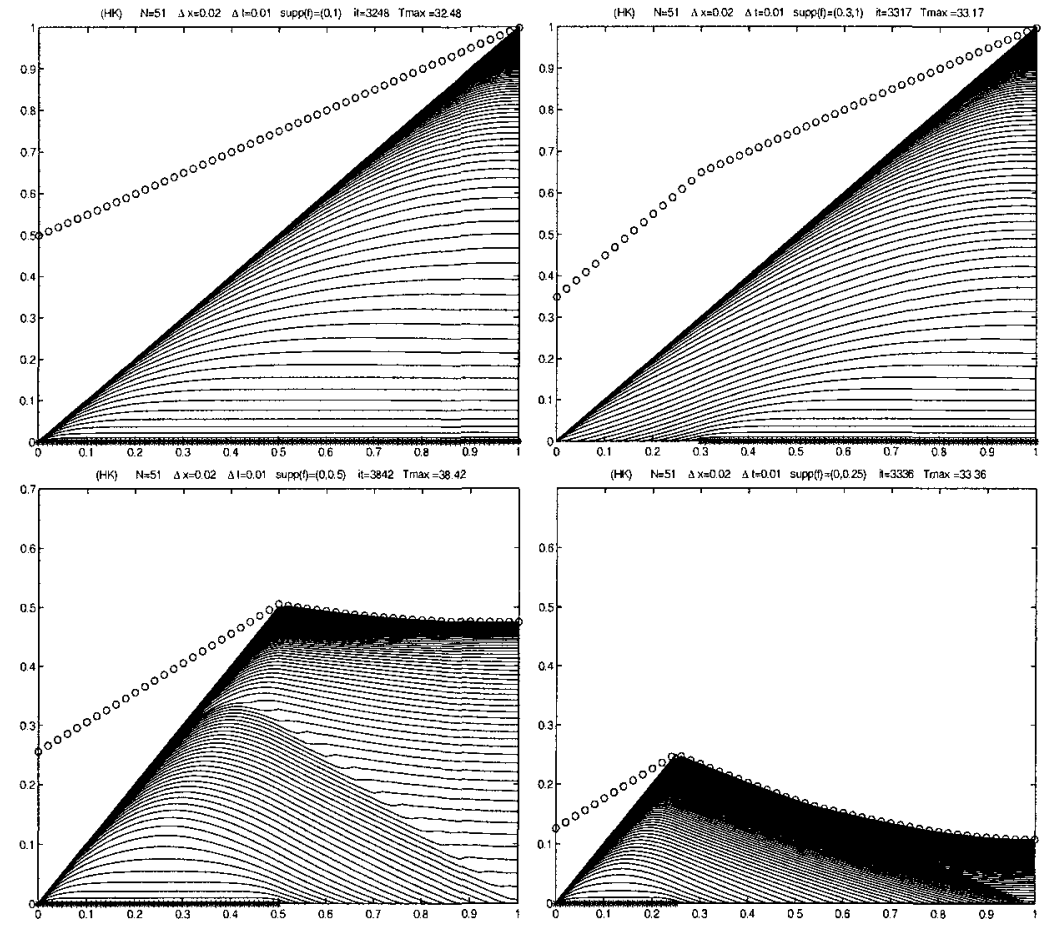

Figure 1. (1D) Growing standing layers on a table closed at the right-hand side, for different source supports $D_{f}$ : a) $[0,1]$; b) $[0.3,1]$; c) $[0,0.5]$; d) $[0,0.25]$.

For $x_{2}<1$ (case (b) of Section 3), we know that the equilibrium solution $u$ in $\left[x_{2}, 1\right]$ is not uniquely determined, so that it can be strongly influenced by the boundary condition at the wall. Our condition (15) in that case becomes equivalent to a zero normal derivative for $u$, as shown in the two examples of Figure 1 (bottom). We can see that the rolling layer is correctly equal to zero in that region. The final value of $u_{N}$ is directly proportional to the intensity of the source and to $x_{2}$.

\subsection{2-dimensional tests}

We have implemented the two-dimensional scheme (17)-(19),(21)-(24),(25) in the case of a constant source term $f$ with $D_{f}=\Omega$, being $\Omega$ the unit square. In the first example $\Gamma_{1}$ coincides with one side of the square $\Omega$ (see Figure 2). In this case there are no singular points on the boundary, and the stationary rolling layer can be proved to be continuous. The scheme produces the correct dynamics and equilibria, even without the gradient condition (25). 

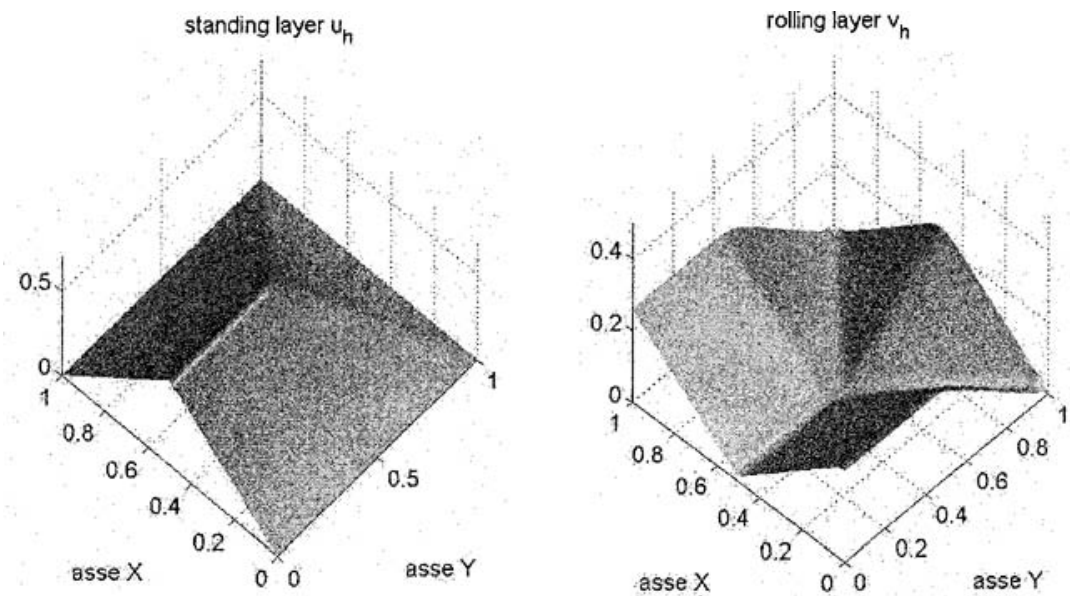

Figure 2. (2D) $f \equiv 0.5, D_{f}=\Omega, N=41: \Gamma_{1}=\{0<x<1, y=0\}$.

In the other three examples (Figures 3,4 and 5) it is possible to see the effect of the boundary singular points. The time iterates $u^{n}$ converges towards the correct distance function from $\Gamma_{0}$ (left), whereas the rolling layer $v^{n}$ very quickly grows around the singular points in the normal direction to the boundary (the black areas in the pictures, where $v$ is shown from above). An equilibrium configuration is always reached, and the zero level set of the final rolling layer is able to detect efficiently the ridge set (in white).
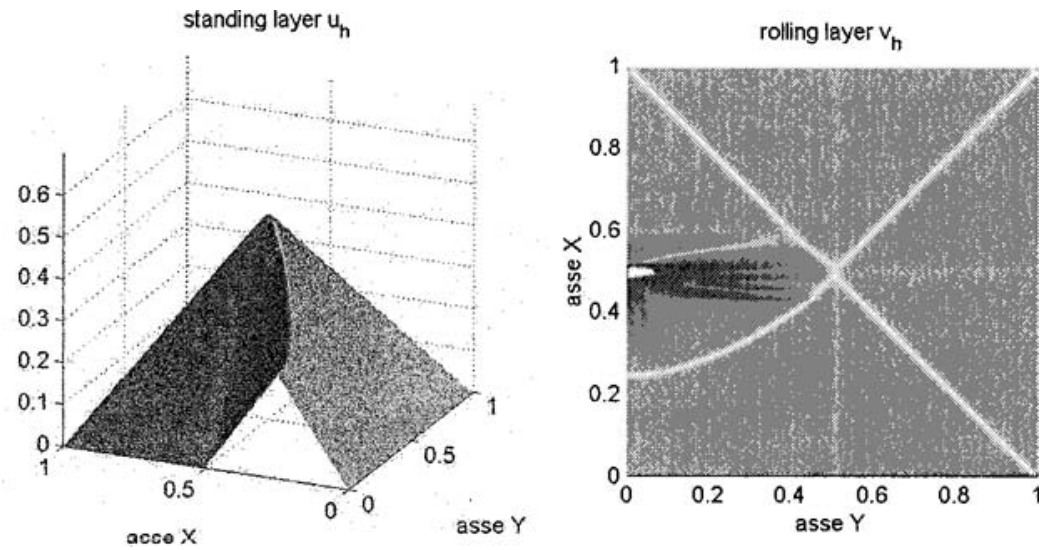

Figure 3. (2D) $f \equiv 0.5, D_{f}=\Omega, N=41: \Gamma_{1}=\{0<x<0.5, y=0\}$. 

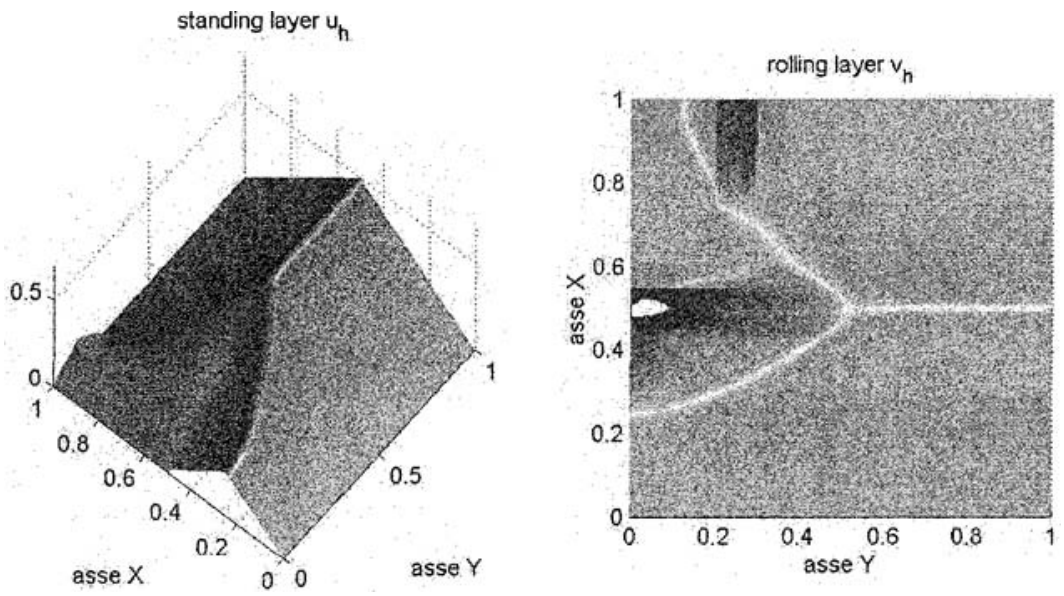

Figure 4. (2D) $f \equiv 0.5, D_{f}=\Omega, N=41: \Gamma_{1}=\{0<x<0.5, y=0\} \cup\{x=0,0<$ $y<0.25\} \cup\{0<x<1, y=1\}$.
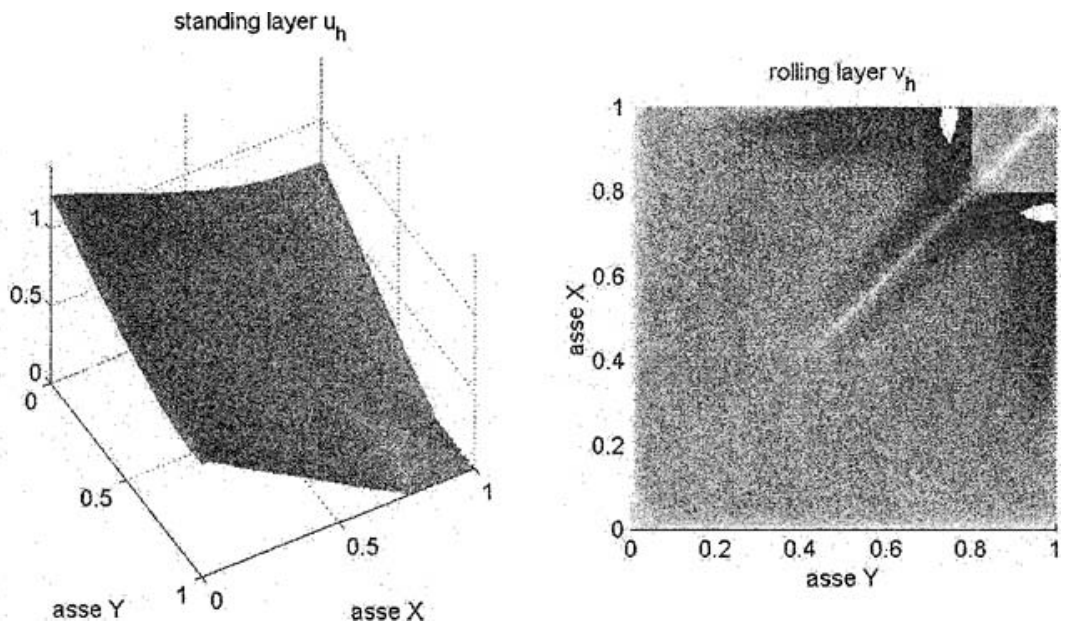

Figure 5. (2D) $f \equiv 0.5, D_{f}=\Omega, N=41: \Gamma_{0}=\{0.75<x<1, y=1\} \cup\{x=$ $1,0.75<y<1\}$. 


\section{Conclusions and perspectives}

We end this paper drawing some partial conclusions on the approximation of the two-layers model that is proposed here. The numerical results show that the scheme is reasonably accurate for the approximation of the stationary standing layer as well as for the approximation of the evolution of $u$ and $v$. The simulation seems to be close to the physical behavior of $u$ and $v$ for all times $t \in[0, T]$. Another interesting feature of the scheme is the fact that it is rather flexible to handle easily the open and the wall problem. The scheme gives also an accurate reconstruction of the ridge without spurious oscillations and this is due to the up-wind discretization of the nonlinear terms of the system.

In the future, we intend to pursue our study completing the analysis of stability in order to obtain a general convergence theorem and attack other problems related to the modelling of granular matter, e.g. the silos and the obstacle problem. Finally, an interesting open problem is to prove an analogue of the representation formula (5) for the wall problem.

\section{References}

[1] G. Aronsson, L.C. Evans and Y. Wu. Fast/slow diffusion and growing sandpiles. J. Diff. Equations 131:304-335, 1996.

[2] P. Cannarsa and P. Cardaliaguet. Representation of equilibrium solutions to the table problem for growing sandpiles. JEMS 6:435-464, 2004.

[3] P.G. de Gennes. Granular matter. In Summer School on Complex Systems, Varenna, Lecture Notes Società Italiana di Fisica 1996.

[4] L.C. Evans, M. Feldman and R.F. Gariepy. Fast/slow diffusion and collapsing sandpiles. J. Diff. Equations 137:166-209, 1997.

[5] M. Falcone and S. Finzi Vita. A finite-difference approximation of a two-layer system for growing sandpiles, Dipartimento di Matematica, Preprint, May 2005 also available at http://cpde.iac.rm.cnr.it/preprint.php.

[6] M. Falcone and S. Finzi Vita. Convergence of a finite-difference approximation of a twolayer system for growing sandpiles, in preparation.

[7] S. Finzi Vita. Numerical simulation of growing sandpiles. In Control Systems: Theory, Numerics and Applications, CSTNA2005. e-published by SISSA, PoS (http://pos.sissa.it), 2005.

[8] K.P. Hadeler and C. Kuttler. Dynamical models for granular matter. Granular Matter 2:9-18, 1999.

[9] K.P. Hadeler, C. Kuttler and I. Gergert. Dirichlet and obstacle problems for granular matter. Preprint, University of Tübingen, 2002.

[10] L. Prigozhin. Variational model of sandpile growth. Euro. J. Appl. Math. 7:225-235, 1996. 